\title{
Current Status and Future Technical Challenges for Tokamak Magnets
}

\section{N. Martovetsky, J. Minervini, K. Okuno, E. Salpietro, O. Filatov}

This article was submitted to $15^{\text {th }}$ Topical meeting on Fusion Technology American Nuclear Society meeting Washington DC, Nov 17-21, 2002

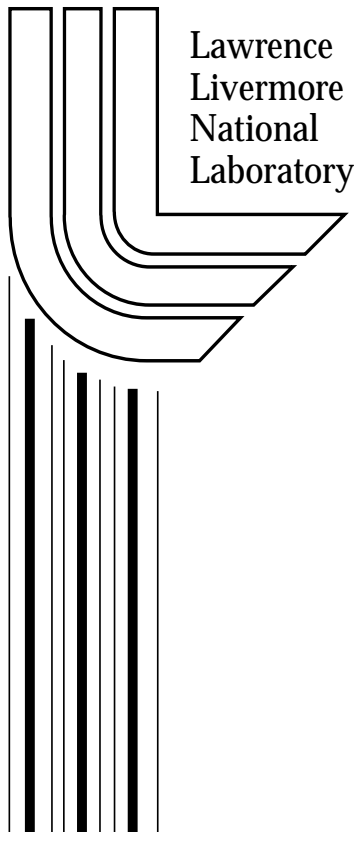

November 11, 2002 


\section{DISCLAIMER}

This document was prepared as an account of work sponsored by an agency of the United States Government. Neither the United States Government nor the University of California nor any of their employees, makes any warranty, express or implied, or assumes any legal liability or responsibility for the accuracy, completeness, or usefulness of any information, apparatus, product, or process disclosed, or represents that its use would not infringe privately owned rights. Reference herein to any specific commercial product, process, or service by trade name, trademark, manufacturer, or otherwise, does not necessarily constitute or imply its endorsement, recommendation, or favoring by the United States Government or the University of California. The views and opinions of authors expressed herein do not necessarily state or reflect those of the United States Government or the University of California, and shall not be used for advertising or product endorsement purposes.

This is a preprint of a paper intended for publication in a journal or proceedings. Since changes may be made before publication, this preprint is made available with the understanding that it will not be cited or reproduced without the permission of the author.

This report has been reproduced directly from the best available copy.

Available electronically at http://www.doc.gov/bridge

Available for a processing fee to U.S. Department of Energy

And its contractors in paper from

U.S. Department of Energy

Office of Scientific and Technical Information

P.O. Box 62

Oak Ridge, TN 37831-0062

Telephone: (865) 576-8401

Facsimile: (865) 576-5728

E-mail: reports@adonis.osti.gov

Available for the sale to the public from

U.S. Department of Commerce

National Technical Information Service

5285 Port Royal Road

Springfield, VA 22161

Telephone: (800) 553-6847

Facsimile: (703) 605-6900

E-mail: orders@ntis.fedworld.gov

Online ordering: http://www.ntis.gov/ordering.htm

OR

Lawrence Livermore National Laboratory

Technical Information Department's Digital Library

http://www.llnl.gov/tid/Library.html 


\title{
CURRENT STATUS AND FUTURE TECHNICAL CHALLENGES FOR TOKAMAK MAGNETS
}

\author{
N. Martovetsky, LLNL, 7000 East Ave, Livermore, CA 94450, USA, \\ martovetsky1@llnl.gov * \\ J. Minervini, MIT PSFC, 185 Albany St., MA 02139, USA, minervini@psfc.mit.edu \\ K. Okuno, JAERI, 801-01, Naka-machi, Naka-gun, Ibaraki-ken, Japan, 311-0193, \\ okunok@itergps.naka.jaeri.go.jp \\ E. Salpietro, EFDA, Boltzmannstrasse 2, D-85748 Garching, Germany, \\ salpiee@ipp.mpg.de \\ O. Filatov, NIIEFA, Sovetsky prospekt, Metallostroi, Saint Petersburg, 189631, \\ Russia, filatov@niiefa.iter.spb.su
}

\begin{abstract}
Magnet technology for fusion in the last decade has been focusing mostly on the development of magnets for tokamaksthe most advanced fusion concept at the moment. The largest and the most complex tokamak under development is ITER. To demonstrate adequate design approaches to large magnets for ITER and to develop industrial capabilities, two large model coils and three insert coils, all using full-scale conductor, were built and tested by the international collaboration during 1994-2002. The status of the magnet technology and directions of future developments are discussed in this paper.
\end{abstract}

Index Terms - Superconducting magnets, Cable-in-Conduit, conductors, losses, superconductor magnets.

\section{INTRODUCTION}

At the moment, the tokamak concept of magnetic plasma confinement is the most advanced direction of research towards burning plasma and the demonstration of a fusion reactor. Other concepts of magnetic confinement, such as stellarators and mirror configurations, have been also employed in plasma research, but the magnet technology for the tokamak has been under development on a much larger scale than for any other concept. For several decades the tokamak concept has been the main focus of fusion research. Plasma parameters, technological approaches and materials developed up to now in tokamak research by far exceed the successes of alternative fusion concepts, and have attracted the most of the funding available for fusion R\&D. Enabling technologies supporting the tokamak concept, in the framework of projects like NET, INTOR, and ITER, machines like TFTR, JET and JT-60, Tore Supra, CMOD, DIIID and others, have achieved impressive progress in all tokamak subsystems and particularly in the magnet systems. The choice for tokamak magnet systems of the future lies clearly with the superconductors, as resistive magnets would require too much electrical power. As soon as superconducting magnet technology was able to meet the requirements for tokamak magnets, it became the leading design choice. First, toroidal field magnets were made superconducting (on Tore Supra and T-15), and later, superconducting Central Solenoid and Poloidal field coils have been developed as well. Impressive progress was made and great experience obtained on the superconducting systems of Tore Supra in France, T-15 in Russia, KSTAR in Korea, HT7U in China, and SST-1 in India, even though the last three machines are still under construction, but have been carrying out intensive R\&D. ITER R\&D has made a very large contribution to the magnet technology for fusion during the last 10 years, particularly through the Model Coil programs. After years of R\&D the leading concept for the conductor for superconducting machines became cable in conduit, introduced by the MIT group led by M. Hoenig. This design combines many of the essential features needed for fusion magnets, such as high mechanical strength, reliable helium containment, favorable conditions for reliable electrical insulation, high stability against electromagnetic and thermal disturbances, and low electromagnetic losses.

A recent intensive US study of burning plasma experiment concepts [1] confirmed that the tokamak is the most viable concept for burning plasma machines at present and for the foreseeable future. All three machines considered for the burning plasma experiment in the nearest future: ITER, FIRE and Ignitor are tokamaks.

This paper presents the status of magnet technology for tokamaks, mostly superconducting, and the directions of future R\&D efforts in magnets.

\footnotetext{
* This work wss performed under the auspice of the US DOE by the UC, LLNL under Contract No. W-7405-Eng-48.
} 


\section{MAIN FEATURES OF TOKAMAK MAGNETS}

The magnet system of a tokamak is one of the most important and expensive components of the machine. The 2001 ITER magnet system cost, for example, without auxiliary systems, represents about $25 \%$ of the total cost of the machine [2]. Therefore, in any of the machines under design or construction, very close attention is paid to magnet system issues.

Tokamak magnets provide the following functions:

Produce the main toroidal field for plasma confinement and insulation from the vacuum chamber walls

Initiate the plasma current for ohmic heating

Generate the poloidal field for plasma control and shaping

Provide correction fields for plasma control and for cancellation of field errors.

These functions are produced by different magnet systems: a toroidal field system, a central solenoid, a poloidal field system and a system of correction coils. The toroidal and poloidal coils are much more challenging; in this paper we concentrate our attention on them. Fig.1 shows location of the main coils in ITER tokamak [3].

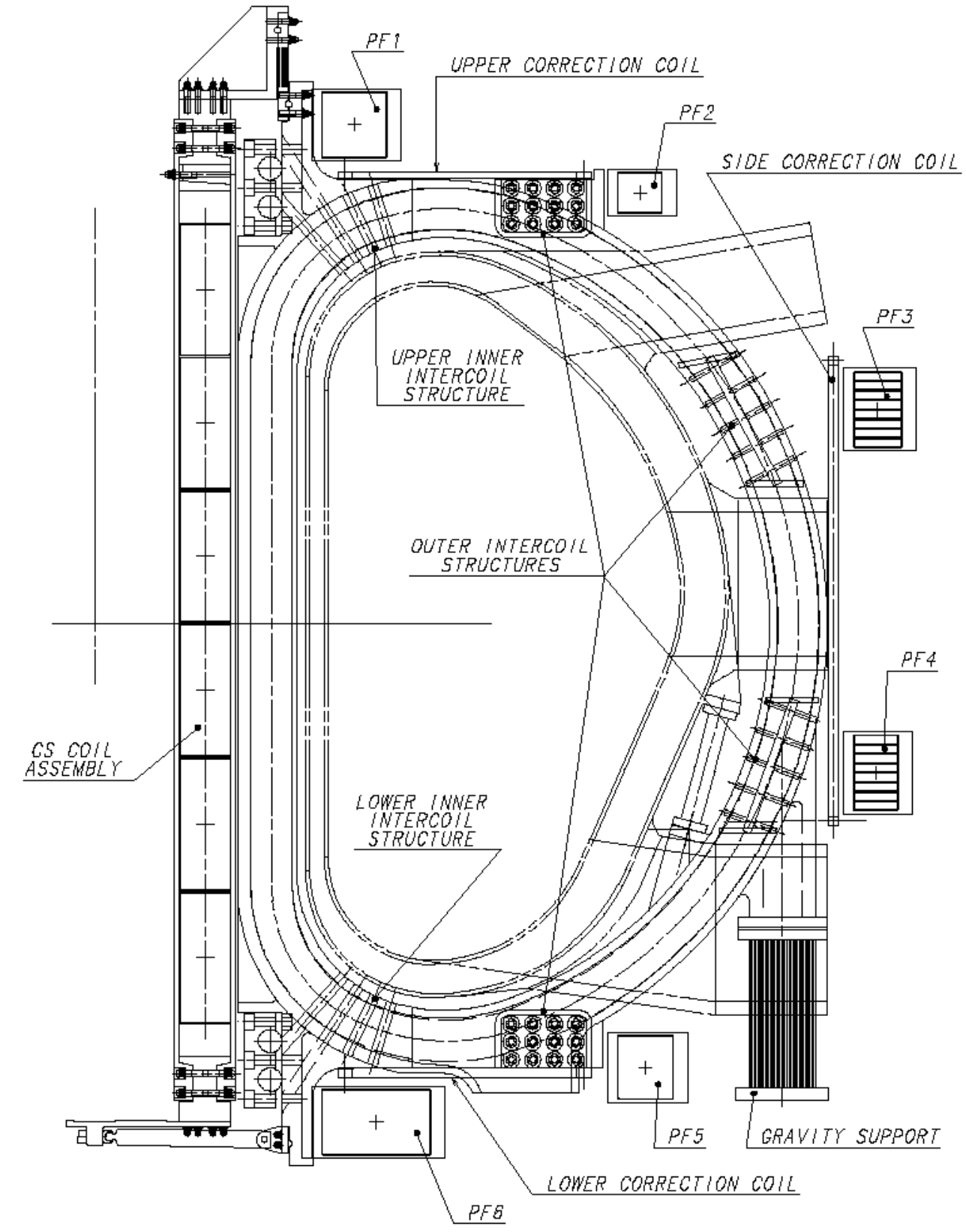

Fig. 1. Configuration of the ITER magnets 
A. Toroidal Field Magnet Subsystem

The toroidal field (TF) coil system is the largest magnet system of the tokamak. The TF system generates a high toroidal DC field for plasma confinement. The highest field strength is always desired for plasma physics, but, of course, this is limited by compromises in dimensions, stresses, material properties and cost. The TF coils see relatively small varying field, but the TF system stores a very large amount of energy and generates enormous forces. The toroidal field creates a very large force towards the machine center, which needs to be reacted at the interfaces between the toroidal coils inner legs (by so-called wedging) and in some designs all or part of the centering forces are reacted by a central bucking structure or by the central solenoid. The outer leg of the toroidal coils experiences an outward force, and the distribution of the volumetric force along the length of the outer leg can generate very high bending stresses. To solve this problem, the geometry of the outer leg is designed in a special shape to eliminate the bending moment, according to an algorithm introduced by V. Shafranov, who showed the feasibility of such a design approach for toroidal magnets [4].

Another challenge to the toroidal magnet support system comes from the interference between the poloidal system and the toroidal coils. The fields from the poloidal coils and plasma current generate large forces on the top and bottom portions of the toroidal coils, creating very large out-of-plane forces and overturning moments. To support these forces the toroidal system needs to employ an intercoil structure, which also must break all conductive paths circumferentially to eliminate otherwise large eddy currents. Fig. 2 shows some details of the intercoil structure of the TF coils for ITER, which is designed to handle these forces.

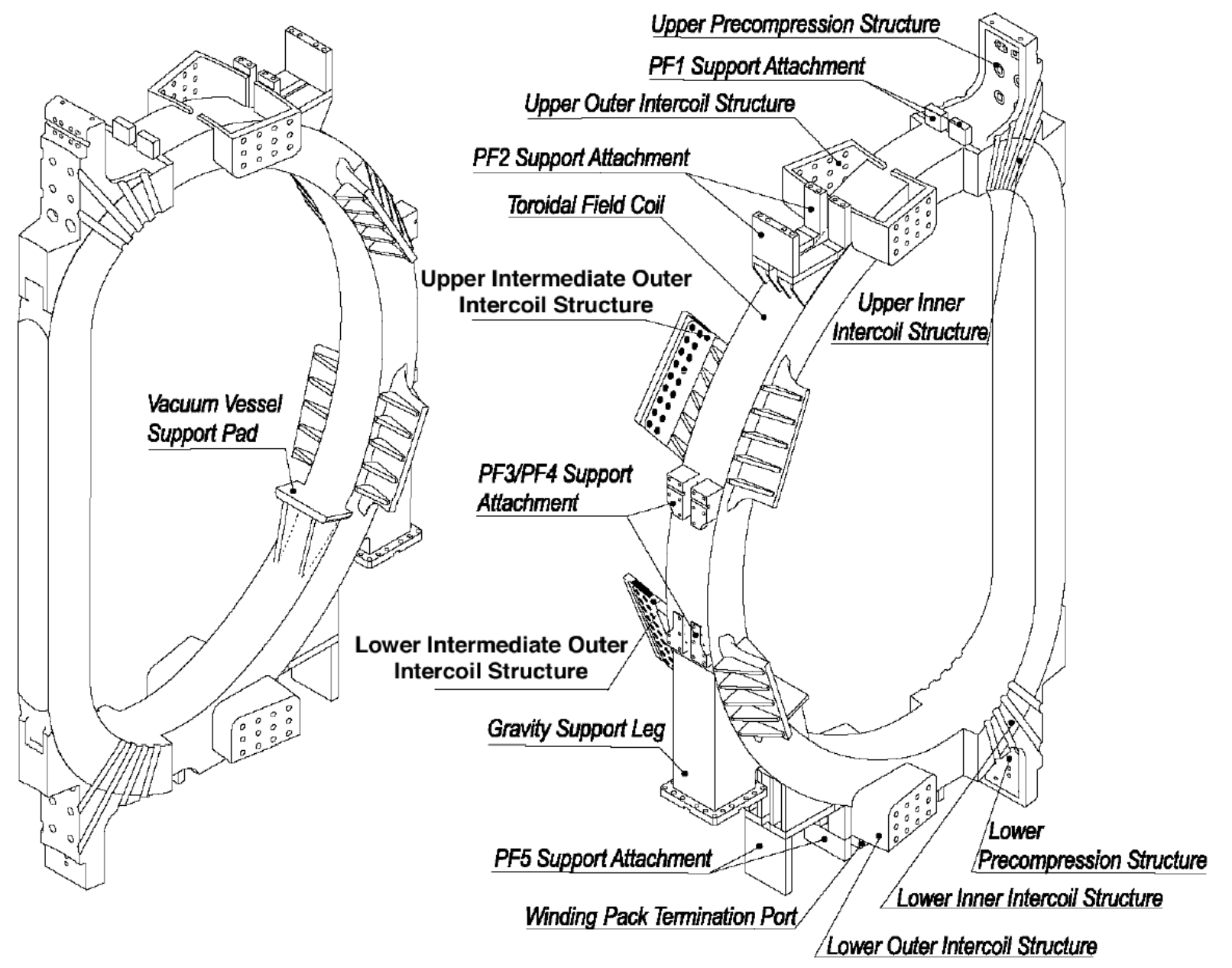

Fig. 2. TF coils structure

The winding pack design of the TF coils in superconducting machines needs to support huge forces in a very limited space and must not expose the conductor to too high compressive stresses, which would inevitably degrade its performance. In small and middle-sized tokamaks, the structural strength of the conductor conduit may be made sufficient to support these forces. But for larger machines, like ITER, the TF coil structure uses radial plates to remove 
the conductor from the load path, so that each individual conductor transfers its force to the structure bypassing the other conductors. Such a structure with a heavy case and radial plates eliminates the conductor's exposure to the huge compressive forces arising from the wedging of the TF coils array. Fig. 3 shows the ITER TF winding pack design, showing radial plates with the embedded conductor inside the massive steel case.

Due to such design of the TF winding pack, the TF coil conductor does not have to be structurally very strong, which makes conductor fabrication and winding much easier. The cross section of the TF conductor used for the TF Insert is shown in Fig. 4. It shows about one thousand strands cabled in a multi stage cable inserted in a relatively thin wall conduit. The primary purpose of the conduit is to serve as a helium container. The central hole in the conductor serves to reduce the hydraulic impedance and corresponding pump work of the helium pumps, which represents a significant fraction of the cryogenic load on the tokamak refrigerator.

The major technical challenges of the toroidal coil system comes from a very large number of conflicting requirements to generate the highest possible field (since fusion power is proportional to $\mathrm{B}^{4}$ ), and to support very large forces in a very wide operating range dictated by physics scenarios, in a very limited space. Since the system is assembled from many elements of a very large size, the fabrication, tolerances, assembly, access and maintenance issues are very demanding. The TF magnet system for ITER stores energy equivalent to approximately $10 \mathrm{t}$ of TNT, which must be safely evacuated from the magnet or evenly distributed in the event of a quench. Currently the protection is assured by evacuating most of the stored energy onto dump resistors and by providing enough copper in the conductor cross section to limit the hot spot temperature to $150 \mathrm{~K}$ in the event of a quench.

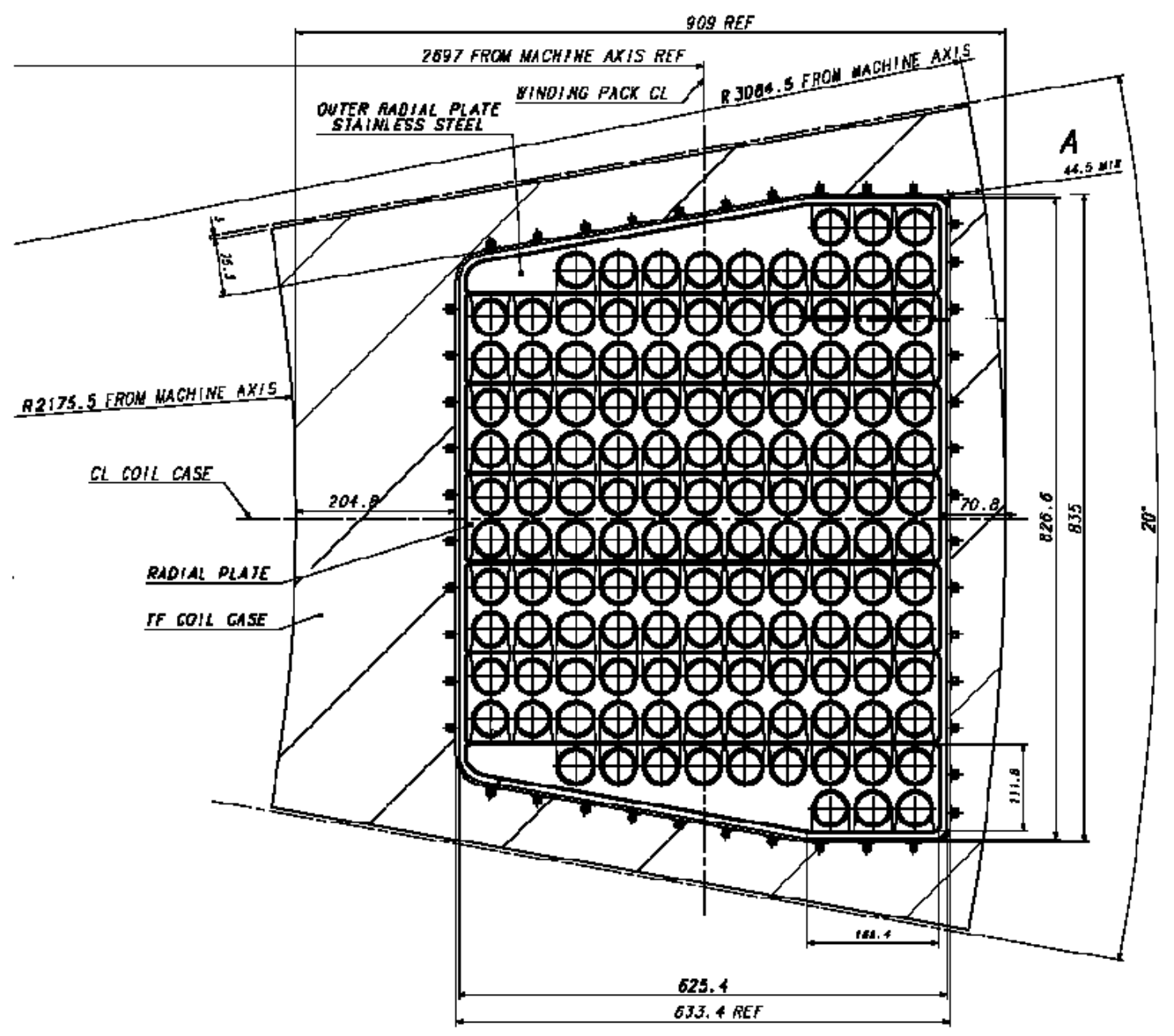

Fig. 3. TF winding pack design at the inner leg

From Fig. 3 and 4 one can see that there is a significant amount of structural steel, copper, helium and superconductor in the TF coil cross section and improving just one of the elements in the coil will not significantly improve the performance of the winding pack (although, the cost of the TF coils can be reduced significantly if the cost and/or 
performance of the superconductor could be significantly improved). The ITER 2001 final design is optimized for $12 \mathrm{~T}$ peak field, $5 \mathrm{~T}$ field on the plasma center, resulting from a reasonable balance between 316LN structural steel strength, modern properties of the Nb3Sn superconductor and reliable and conservative approach to the amount of copper in the conductor for protection and stabilization.

To significantly improve the performance of tokamak TF coils in the future we need a complex of advances: improve significantly the superconducting properties of the conductor to increase the engineering current density in the conductor, reduce the amount of copper by finding more efficient means of coil protection, develop more reliable insulation systems which would allow higher voltages at the dump, which in turn would require less copper in the conductor, and develop stronger and tougher structural materials which could withstand higher stresses and survive a significantly larger number of cycles in operation. Special attention will be paid to the compatibility of the structural materials with the properties of superconductors such as thermal expansion and with fabrication and operation steps such as heat treatment cycle and cooldown. Reduction of copper in the winding pack may eventually lead to a lower stability; however there is evidence [5] that even a reduced amount of copper may be sufficient for TF coils stability, therefore an improvement in this area looks very promising.

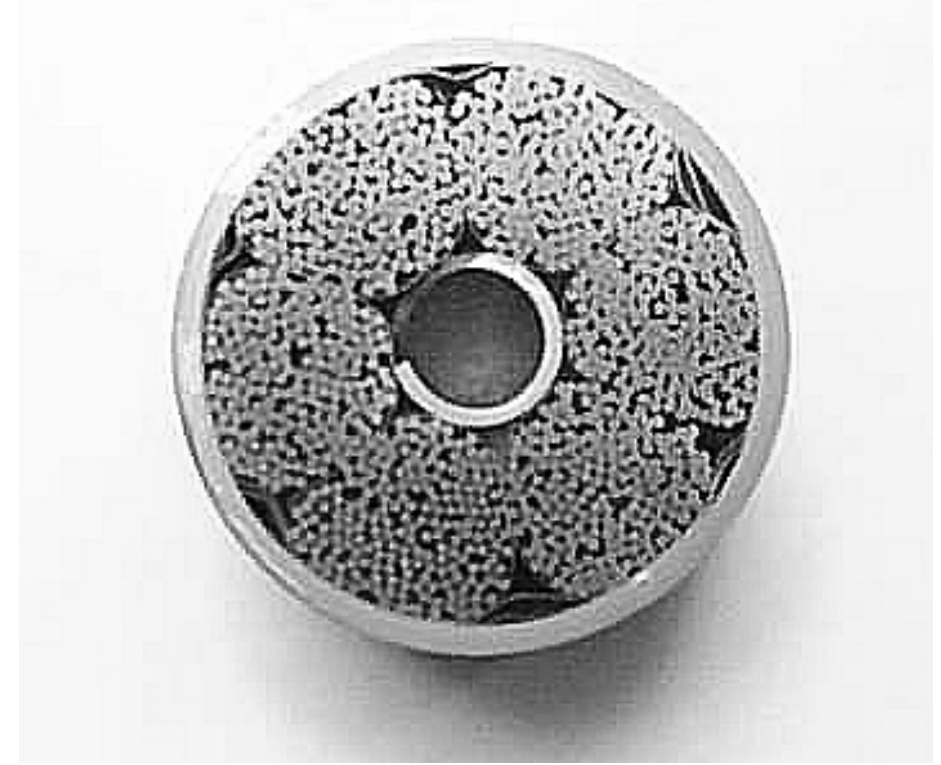

Fig. 4. Cross section of a TF Insert conductor, 43-mm diameter, 1152 strands.

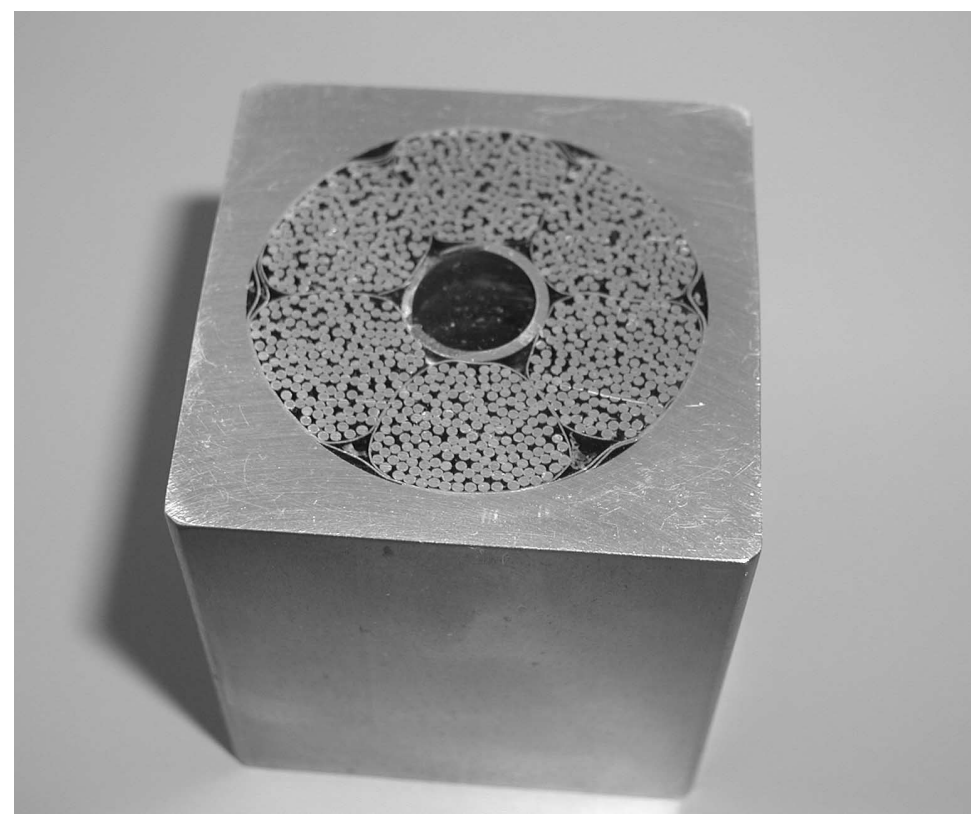

Fig. 5. A piece of a CSMC conductor, $51.2 \mathrm{~mm}$ square, 1152 strands in a heavy wall conduit 
B. Poloidal Field Subsystem, including the Central Solenoid

The technical requirements for the central solenoid (CS) and poloidal system are quite different from the TF system requirements. The CS main purpose is to ignite and burn plasma. That implies that the CS should have high stability against fast field variation at the initiation and generate enough magnetic flux to control the plasma burning.

Therefore, the structure to support electromagnetic loads in the CS should not have circumferentially closed paths, but must still support very substantial hoop and axially compressive forces. That results in the selection of a conductor with a robust cross section, which serves as a distributed structure. Fig. 5 shows the CS conductor for the ITER 1998 final design.

The high flux requirement results in a high peak field requirement for the CS: for the ITER design it is 13.5 T. Thus, with less complicated structural requirements in comparison with the TF magnet subsystem, the CS subsystem's most difficult task is to provide enough stability and cooling capacity to withstand fast field variations. Therefore the main focus of the CS has been on conductor development and characterization.

Another potential problem area in the CS is the insulation. Hoop and axial stress and dissimilar thermal and mechanical properties of the insulation and conductors create very high stresses in the insulation, which in some cases become the CS design drivers. Stronger insulation systems, with higher radiation toughness will be required for reactors of the future.

The PF coils of the tokamak have very large size but modest magnetic field requirements. The choice of superconductor for the PF for all superconducting tokamaks so far was NbTi. The main effort of the R\&D is in making sure that relatively low temperature margins of $\mathrm{NbTi}$ and long hydraulic lengths are sufficient to provide an adequate stability in such large current conductors, which need to carry about $50 \mathrm{kA}$ peak current.

\section{ITER MODEL COIL R\&D EFFORT}

To develop methods of CS and TF coil fabrication, including all components and to demonstrate feasibility and industry readiness, two projects on magnet technology were undertaken by the ITER collaboration. In the CS Model Coil project, a large CS Model Coil and three inserts with the full size conductor were built and tested [6-8] in the background field of the CSMC. The CSMC is more than $100 \mathrm{t}$ in mass and it stores $650 \mathrm{MJ}$ at $46 \mathrm{kA}$ and $13 \mathrm{~T}$ peak field - the largest pulsed superconducting magnet ever built. The three Inserts were a CS Insert and an $\mathrm{Nb}_{3} \mathrm{Al}$ Insert built by Japan, and a TF Insert built by Russia. The CSMC and the CS Insert used the conductor shown in Fig.5 with the conduit made of superalloy Incoloy 908, developed by MIT to match the coefficient of thermal expansion (CTE) of the superconductor $\mathrm{Nb}_{3} \mathrm{Sn}$. Using this material instead of stainless steel increases the current carrying capacity of the superconducting filaments by $30-50 \%$ in 12 T, which was confirmed in many small scale conductors. Also, Incoloy 908 has excellent mechanical properties.

The TF Insert was built with Ti conduit to see the performance of the well-instrumented thin jacket conductor in fields up to $13 \mathrm{~T}$ and also to find out how much advantage could be gained by using the low CTE conduit in the stainless steel structure: a configuration relevant to the TF coils.

The $\mathrm{Nb}_{3} \mathrm{Al}$ insert was designed and built to explore the alternative conductor, but also, being less sensitive to the strain, the $\mathrm{Nb}_{3} \mathrm{Al}$ Insert helped to understand some strain related phenomena observed in the $\mathrm{Nb}_{3} \mathrm{Sn}$ conductors.

The TF Model Coil Project [9] also used the full size conductor, similar to shown in Fig.4 in the oval coil configuration with radial plates, simulating the ITER TF coils. The TFMC was tested in "stand alone" configuration and is being tested as of fall of 2002 in the background of the LCT magnet to bring the operating point closer to what the conductor will see in ITER operation, although, as with any modeling, some extrapolation will be necessary to assess the performance in much higher operating field of ITER.

The focus of these two projects reflected the requirements of the TF and CS systems. The TF Model Coil was more complicated in fabrication and was mostly focused on the fabrication issues, although testing the TFMC provided very valuable information for the ITER design. The CSMC program focused on gaining the maximum possible information about the performance of the conductors, especially in the heavily instrumented inserts, under different conditions simulating ITER and under much more severe conditions to determine the operational margins.

From the point of view of conductor performance, the following are the most important issues studied in the Model Coil testing: DC performance, AC losses, ramp rate sensitivity, and performance of the joints. We will discuss the results in some detail in this paper. There were many other issues studied relevant to the tokamak magnets, such as quench detection, normal zone propagation, and hydraulic characteristics, but we will not discuss them here.

\section{A. DC Performance and Needs for High Performance Superconductor}

The DC performance shows how much of the potential current carrying capacity of the cable is realized in the magnet. If we know the critical current of a strand, which is used in the CICC, say, in 13 T and at $6 \mathrm{~K}$, we expect to see that 1152 
strand cable in $13 \mathrm{~T}$ and at $6 \mathrm{~K}$ should carry 1152 times the critical current of the strand at the same level of electrical field and stress conditions. If the achieved CICC current is smaller, it is called degradation, and to overcome degradation, one has to choose a safety margin, which would make up for the loss of current carrying capacity. This is of course, a simplified picture: since strain conditions in the CICC and the strand are usually quite different, the selffield of the CICC is very strong, which prevents the conductor from having a uniform magnetic field across the whole cross section. The electrical field in the CICC strands cannot be as uniform as in individual strand due to twisting in the cable. These and several other effects make comparison of the strand performance to the CICC performance a very complicated task. However the analysts are trying take into consideration all these details when comparisons are made.

Fig. 6 summarizes the performance of the magnets tested in the ITER Model Coil program. All data are referred to the $12 \mathrm{~T}$ operation point using correlations obtained in the post-test analyses of all these tests [8]. The dashed lines show the performance of the original strands used in the magnets and the horizontal arrows from these dashed lines to the solid lines represent the degradation of the CICC. The $\mathrm{Nb}_{3} \mathrm{Al} \mathrm{CICC}$ did not experience any degradation; therefore the strand and the CICC performance coincide. The TFMC CICC performance is calculated assuming $-0.7 \%$ compression, in this sense the TFMC degradation results entirely from the natural mismatch between the strand and the stainless steel conduit which is expected, in contrast to the other $\mathrm{Nb}_{3} \mathrm{Sn}$ magnets (CS Insert and TF Insert), where degradation is not fully explained.

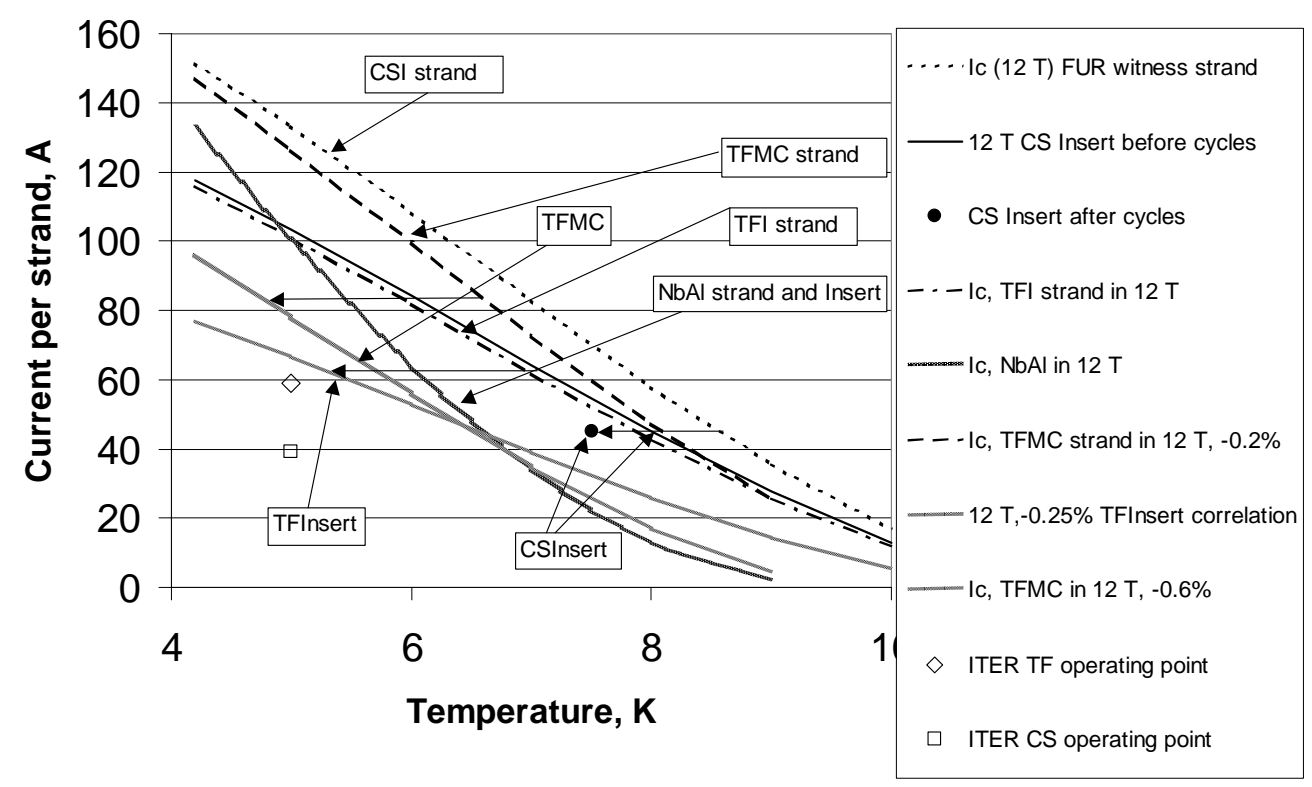

Fig.6. Summary of the Ic measured in the ITER Model Coil projects

The ITER operating points in the 2001 FDR correspond to a thinner strand with higher jc, but the Ic requirement of $12 \mathrm{~T}$ remains very close to the Ic requirement of the grade 2 strands used in the Model Coil program. That makes the comparison of the tested conductors to the new ITER requirements sensible. Fig. 6 shows that with the observed degradation the operating point is achievable, but the safety margin might be somewhat lower than is considered conservative, especially in the in the TF coils.

It is seen that in terms of Tcs margin, the $\mathrm{Nb}_{3} \mathrm{Al}$ CICC is significantly better than the TFMC and TF Inserts at the ITER TF operating current, but lower than the CS Insert CICC despite degradation of the latter. In other words while the $\mathrm{Nb}_{3} \mathrm{Al}$ strand is a very good predictor of the CICC performance, the best $\mathrm{Nb}_{3} \mathrm{Sn}$ materials in low CTE conduit exceed the performance of the $\mathrm{Nb}_{3} \mathrm{Al}$ strands even after degradation of the $\mathrm{Nb}_{3} \mathrm{Sn}$. Recent progress in $\mathrm{Nb}_{3} \mathrm{Sn}$ strands makes them even more superior [10]. 


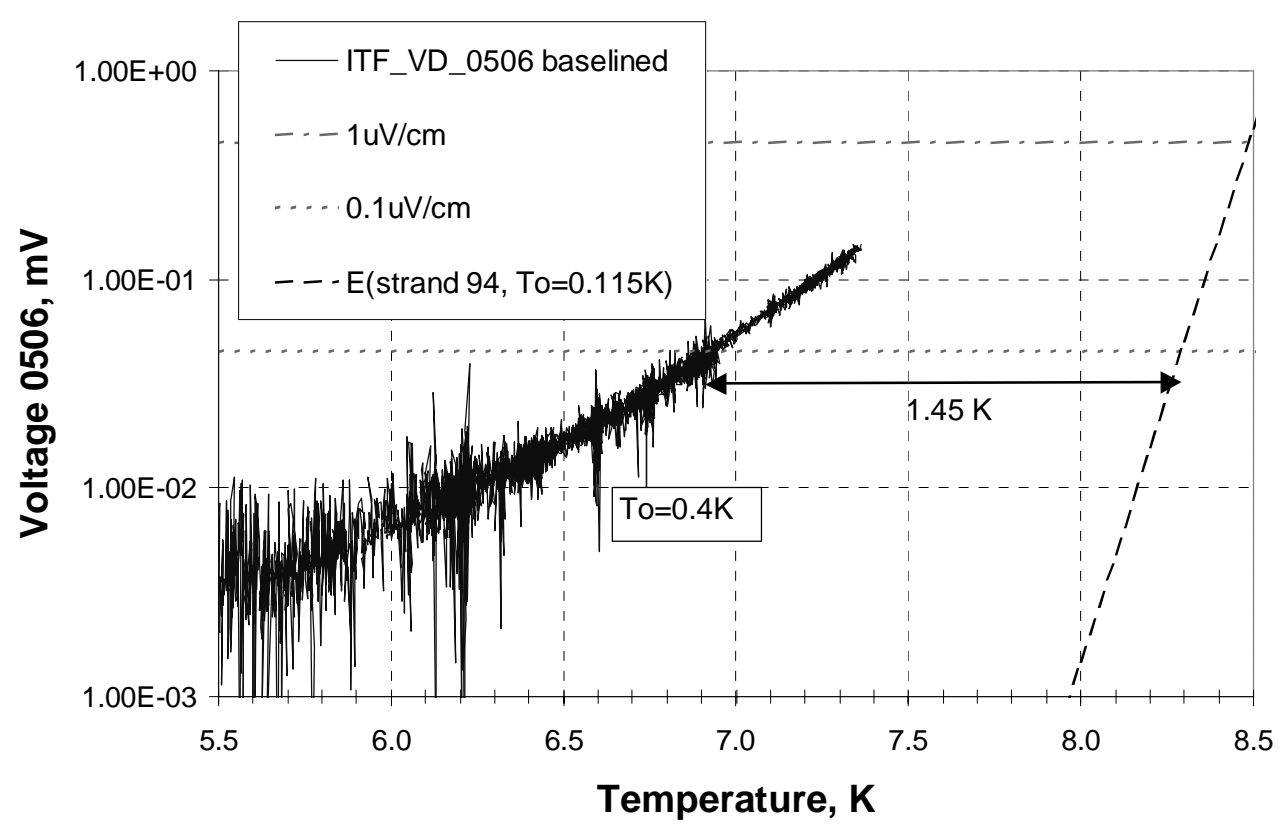

Fig.7. Comparison between transition to normal state in the TF Insert and the strand used in the TF Insert at $46 \mathrm{kA}$ and in $12 \mathrm{~T}$

$\mathrm{Nb}_{3} \mathrm{Sn}$ remains the main candidate material for ITER for several reasons, such as higher performance, lower cost and significantly higher availability. Therefore, the observed degradation of the high current CICC in the CSMC project has become the focus of the magnet technology R\&D effort. This degradation does not constitute a feasibility threat, since it could be compensated by a higher performance strand, already available from industry, or by putting more of the superconducting strands into the cable, but there is a significant cost incentive to find out the reasons and mitigation to this phenomenon.

Another result obtained on the DC performance of the Model Coils is the observation of a broad transition to the normal state in the cable. The broadness of the transition is defined by the electrical field growth rate versus current or temperature change. This broadening of the transition was first reported on T-15 and later on the Westinghouse LCT coils, which were fabricated using "reactand-wind" technology. In this approach, the conductor is heat treated and the $\mathrm{Nb}_{3} \mathrm{Sn}$ is formed, after which the conductor is insulated and wound into the coils. Although all precautions are made not to break or damage the brittle $\mathrm{Nb}_{3} \mathrm{Sn}$ superconductor during fabrication, in practice it turned out to be difficult or impossible to prevent. That broadening of the transition and degradation was interpreted as mechanical damaging the strands and led the fusion community to abandon this "react-and-wind" technology as too risky for large magnets and to adopt the "wind-react-transfer" technology. The latter reduces the handling of the brittle $\mathrm{Nb}_{3} \mathrm{Sn}$ superconductor after the reaction and thus intends to reduce and eliminate the manufacturing risk of damaging the conductor. In the Model Coils projects the expected advantages of "wind-react-transfer" technology were not completely realized. The exact cause of this is still not clear, although the TFMC did seem to realize the performance of the strand with about expected degradation caused by the stainless steel conduit compression on the cable.

Fig. 7 shows a typical transition to the normal zone in one of the model coils in semi-logarithmic coordinates compared to the transition of the strand. The cable develops an electrical field sooner than the strand and the voltage growth is more gradual. Both phenomena are undesirable for operation, since to suppress the heat generation one has to be significantly below "critical" current sharing temperature or critical current, which decreases effective current density. It is seen also that the loss of critical properties in the cable cannot be expressed in just one number: rather it depends on the voltage level. The change of the transition in the CICC in comparison with the strand has been under detailed investigation in the Model Coil projects and in smaller supporting projects, and it has been established that this phenomenon is most likely stress related, since it was not observed in the stress insensitive $\mathrm{Nb}_{3} \mathrm{Al}$ Insert [8] and NbTi samples [11]. But it is still not clear how much of the degradation is associated with the electromagnetic loading, and how much comes from fabrication, handling and cooldown stresses. It is also unclear how degradation could be avoided or reduced. This investigation is underway.

One of the ways to mitigate the degradation is to provide a larger margin in the CICC. This can be done in several ways. First, the operating temperature could be reduced by, say, $1 \mathrm{~K}$ with some increase of the refrigerator cost. It is well within existing technology 
and it might be a well-justified and economical solution. Another way to increase the margin is to use a higher performance strand, which is also feasible with existing technology. Recent developments in $\mathrm{Nb}_{3} \mathrm{Sn}$ strand have shown impressive progress with jc (12T, $4.2 \mathrm{~K}$ ) approaching $3000 \mathrm{~A} / \mathrm{mm}^{2}$ [10] in the filament area of the internal tin strands, although at the expense of high losses. The model coil program, started in 1992 , specified the jc $(12 \mathrm{~T}, 4.2 \mathrm{~K})$ to be $700 \mathrm{~A} / \mathrm{mm} 2$ for the high loss strands and $550 \mathrm{~A} / \mathrm{mm} 2$ for the low loss strands. Thus, mitigation of the degradation problem for the nearest future machines like ITER does not constitute a materials problem, rather the challenges lie in the area of establishing adequate industrial capacity and cost effective processes. The total world production of $\mathrm{Nb}_{3} \mathrm{Sn}$ strand is currently about 1-2 t/year [12], while to support a reasonable construction schedule for ITER it should be about 150-200 t/year [13].

There are two main technologies to make an $\mathrm{Nb}_{3} \mathrm{Sn}$ strand - a bronze route and an internal tin process. The bronze route process can produce at present a strand with jc $(12 \mathrm{~T}, 4.2 \mathrm{~K})$ up to $600-700 \mathrm{~A} / \mathrm{mm}^{2}$ in the non-copper area with a well-established $14 \%$ Sn content bronze, and $700-800 \mathrm{~A} / \mathrm{mm}^{2}$ with a recently developed $16 \%$ Sn bronze. This may not be sufficient to meet the needs of a machine like ITER [14]. The internal tin process can significantly exceed that performance because in the internal tin process it is possible to have higher $\mathrm{Nb}$ and $\mathrm{Sn}$ content in the strand. However most of the world production capacity is associated with the bronze technology, and establishing an industrial base with sufficient capacity to produce the strand with higher critical current is an important task for magnet technology. The ITER community has already developed plans to realize this goal [15].

There is significant progress being made in HTC superconductors, especially with Bi-2212 strand, which in CICC form can be made to carry tens of kA [16]. Due to its higher critical field, this material is superior to $\mathrm{Nb}_{3} \mathrm{Sn}$ at some field, the so called "crossover field". At present, this field is about 13-15 T [10], depending on the definition of "filament" area and on how much of this area can be practically incorporated in the strand, since the technology of the Bi-2212 strand requires a significant amount of silver in the cross section. It is expected that in the future the technology will be developed to make HTC superconductors more powerful and, hopefully, more cost competitive. If so, the next generation tokamak after ITER may use HTC technology, either in the traditional CICC form, or in a more advanced form, like YBCO coated plates [17]. The latter could make possible 16-20T peak fields in superconducting tokamaks, which would allow significant improvement in plasma burning conditions.

\section{B. AC Losses}

The AC losses in the conductors represent several threats to serviceability. First, it is an additional cryogenic load on the refrigeration system. Second, high losses increase the operating temperature within the CICC and thus reduce the temperature margin in the CICC, which may require an additional amount of superconductor or cooling. Third, the large effective diameter of the filaments in the strands, which are responsible for high hysteresis losses in the strand, may initiate a flux jump in the strands, which can cause instabilities in the strand and even quench in the whole cable.

Therefore it is important to keep losses as low as practical. There are two sources of losses in the CICC - intrastrand losses and interstrand or cabling losses. The intrastrand losses are determined by the strand design, while the cabling pattern and the interstrand resistances determine the cabling losses. The strand hysteresis losses are related to the noncopper critical densities. In the high $\mathrm{Nb}_{3} \mathrm{Sn}$ content strands the interfilament spaces are small and the filaments are coupled through superconducting bridges, therefore although the filaments are 2-5 micrometers thick, electromagnetically they behave as 50-100 micrometer thick clusters, and hysteresis losses just due to the dimensions become proportionally higher. Having both high jc and low losses is a technical challenge, but the leading strand manufacturers supported by fusion have made significant progress lately and high energy physics R\&D efforts [10]. The Model Coil tests showed that there was a significant margin in the TF conductor allowable losses. This follows from the fact that the $\mathrm{Nb}_{3} \mathrm{Al}$ insert, having order of magnitude higher hysteresis losses and significantly higher coupling losses, met all the TF Coil scenarios without problems other than increased refrigeration load. However, the CS Insert operations under initiation conditions or large swing field variations were limited by heat generation in the conductor due to losses. Therefore, losses can be the limiting operating factor under some conditions and, obviously, more effort is required in this area for further improvement.

\section{Ramp Rate Sensitivity}

The ramp rate sensitivity is a phenomenon in which conductors quench at much lower currents under varying field/current conditions than are achievable at a slow charge rate. Ramp rate sensitivity is a potential problem for tokamak magnets, especially for the central solenoid, which must be charged in a reasonably short time, initiate the plasma, and withstand plasma disruptions. In other words, it must withstand appreciable pulsed fields. Ramp rate sensitivity was observed and studied in the DPC program [18] and, since then, has been observed in many CICC. Numerous studies of this phenomenon have revealed that the major reason for this phenomenon is a nonuniform current distribution resulting from non-ideal distribution of the strands in the cable, making loops with different 
inductances, and from nonuniform resistances between strands in the cable, including nonuniform resistances in the joints. It is very difficult to suppress this effect in any conductors, especially in the CICC where there is not much control over the geometrical locations of individual strands and individual contacts between the strands. Even highly geometrically regular so-called Rutherford conductors - one layer, flattened rectangular cables used in particle accelerators - show this effect due to field inhomogeneity. But field inhomogeneity in the cable is a feature of tokamak magnets to an even larger extent if only because of the higher currents in the conductor and, therefore, the more pronounced self-field effect. It is also much more difficult to control the position of the strands in a cable of CICC, let alone the distribution of the resistances between the strands than in a regular cable. The extremes - fully insulated strands or strands with low resistance are not acceptable solutions either. Insulated strands prevent any current redistribution and that leads to a very poor CICC performance. Low resistive links generate unacceptably high losses.

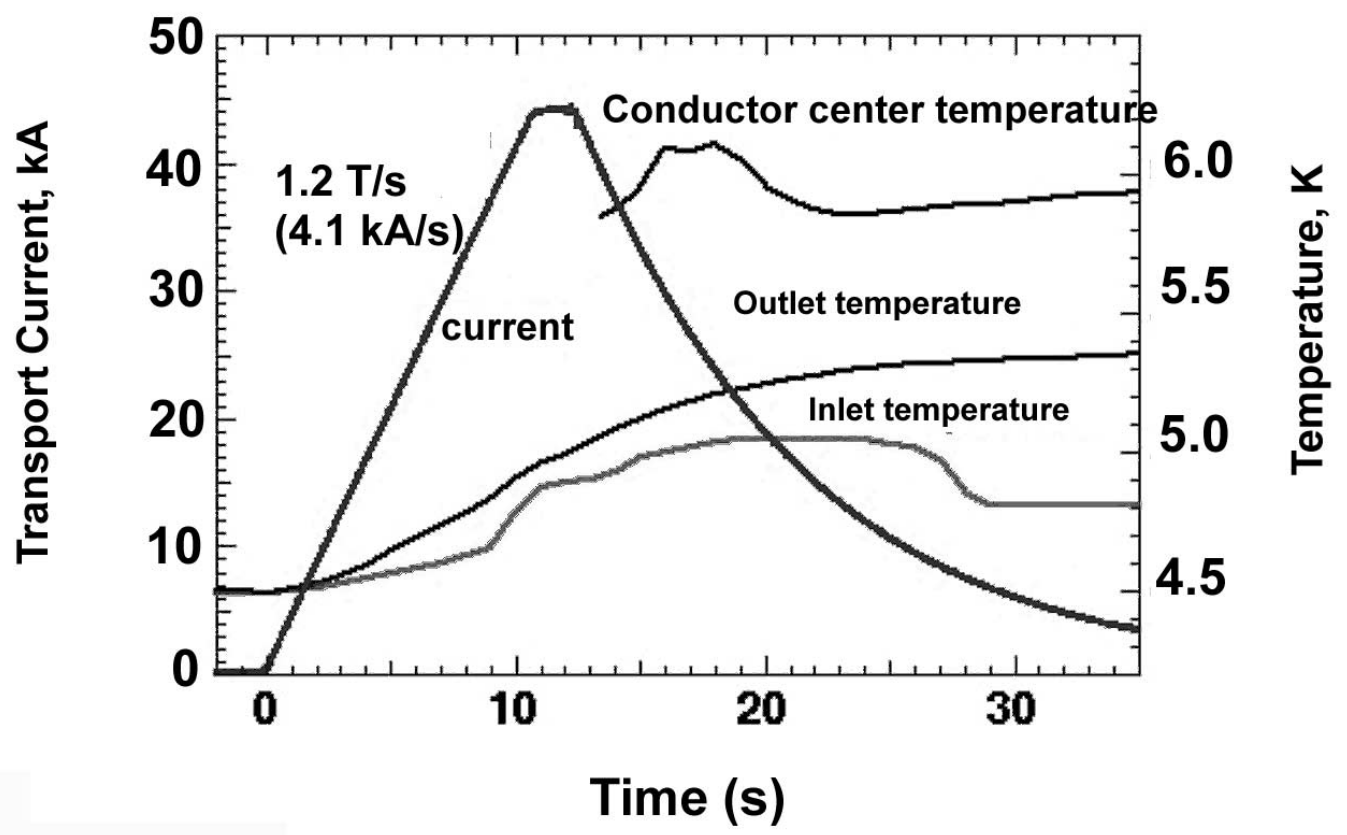

Fig. 8. The $1.2 \mathrm{~T} / \mathrm{s}$ run of the CS Insert and CSMC to $13 \mathrm{~T}$ with no quench.

From studies over the last 10 years, some lessons have been learned and recommendations developed, which help design conductors with more or less low sensitivity to varying magnetic field mostly by reducing the current density in the strands and adding more copper for stabilization. Still the predictive power of these rules is low and by no means do they represent design criteria or laws of nature. Therefore experimental verification of the ramp rate sensitivity is a must for any challenging tokamak system.

The CSMC and the CS Insert magnets were designed with significantly higher safety margins than the R\&D DPC magnets in late 1980-s, where this effect was more pronounced. The Model Coil tests with the CS Insert showed that the ramp rate sensitivity is sufficient to run ITER relevant scenarios with a big margin. Fig. 8 shows the successful run of the CSMC in series with the CS Insert to $13 \mathrm{~T}$ with a rate of $1.2 \mathrm{~T}$, while the requirement was to sustain a $2 \mathrm{~T}$ drop at $1.2 \mathrm{~T} / \mathrm{s}$ rate. The deviation between the currents achievable in a fast ramp and under DC conditions becomes noticeable at $\mathrm{dB} / \mathrm{dt}$ values over $1.2 \mathrm{~T} / \mathrm{s}$ in the CS Insert and above $0.6 \mathrm{~T} / \mathrm{s}$ in the CSMC [6]. Therefore if requirements for tokamak magnets continue to grow, the problem of ramp rate sensitivity will have to be explored in greater detail, and more effective means of its suppression should be found.

D. Joint Performance

The joints are among the most critical elements of tokamak magnets. The joints serve as helium injection ports, current terminals, and instrumentation attachment points. They operate under very high loads, need to absorb large deflections or displacements due to differential material contraction during cooldown, and are frequently exposed to significant varying fields. In past projects joints were always high-risk areas; therefore requirements for reliability are very high.

The Model Coil program demonstrated that more than one solution is available for low resistance joints (see Fig. 9), depending on requirements, typically 1-2 nOhm at 40-80 kA and up to 4T fields. The TF MC joints developed by the 
EU are designed to be very robust and low resistance joints, but not for a varying field applications due to high AC losses. The CS MC joints developed by the US team are of moderate AC losses and low resistance, able to survive up to $1 \mathrm{~T} / \mathrm{s}$ field swings with amplitudes of $4 \mathrm{~T}$ at $50 \mathrm{kA}$. The butt joints developed by the Japanese team are low resistance joints with the lowest AC losses. However they have somewhat lower temperature margin due to fabrication features and therefore are not desirable for operation in high background fields.

All these designs worked very well in the Model Coils and could be used in the other high current CICC applications. Although the joints did not represent limitations on the coil performance, a significant part of the total losses in the coil was associated with the joints. Therefore in future magnets there will be always a requirement to develop low loss, low resistance, compact and reliable joints.
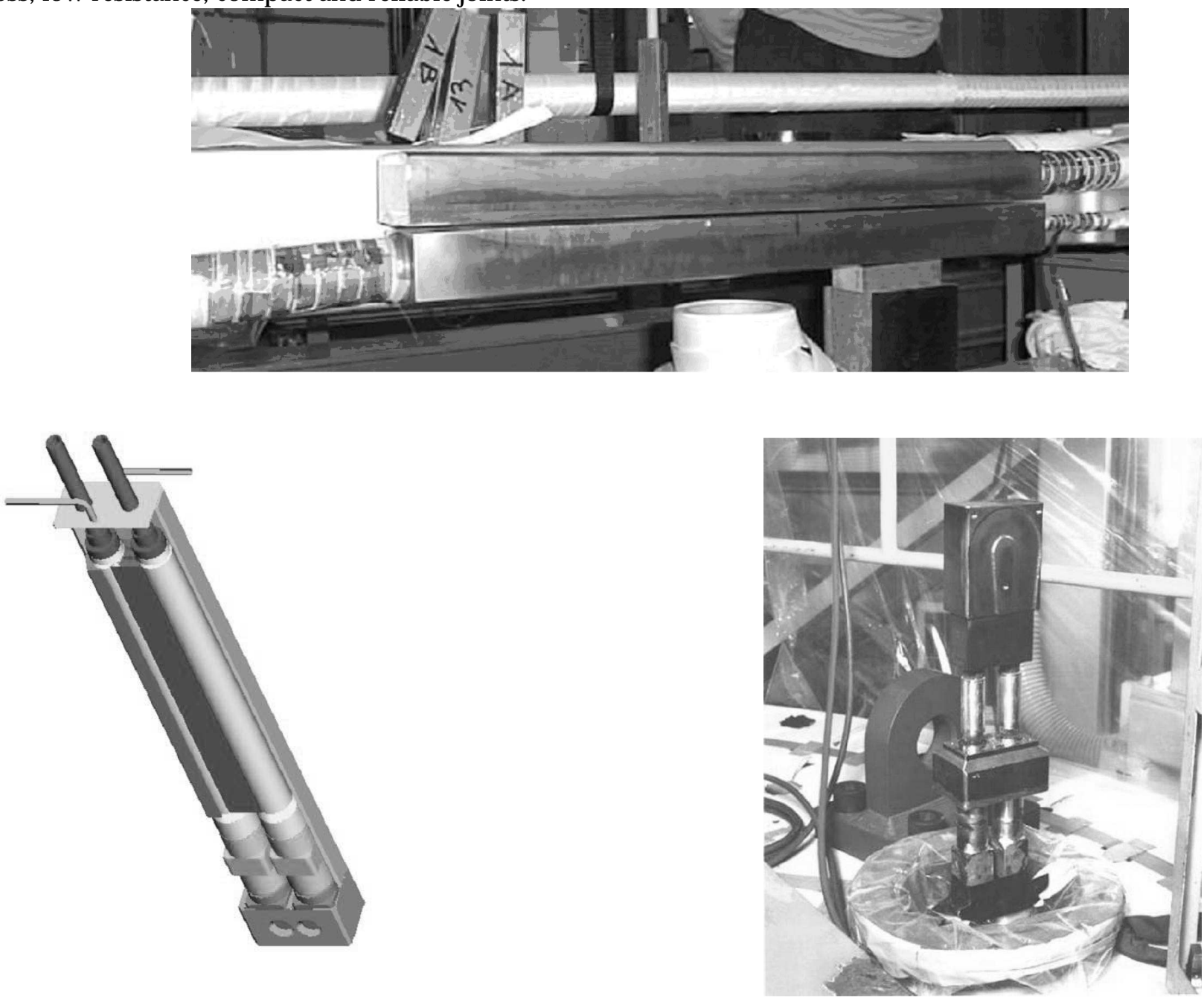

Fig. 9. Types of joints developed for ITER Model Coils: TF Model coil joint (above), CSMC lap joint (lower left) and butt joint (lower right)

\section{CONCLUSION}

Development of tokamak magnets in recent years has significantly enriched large-scale magnet technology. Advanced superconductors have been developed, fabricated and tested. Effective schemes of supporting the operating loads have been created. New materials for conduit, new insulation, and new low friction materials have been developed and proven in operation. Industrial capabilities have been demonstrated or developed for many components of tokamaks, and the feasibility of large superconducting tokamaks has been verified. The nearest future effort in magnet technology is expected to come from the construction of ITER. Building the ITER tokamak magnets will no doubt immensely promote magnet technology in many areas. Development of conductors for CS and TF systems still remains the most critical issue for superconducting tokamak magnets. But to achieve significant further progress in 
future tokamak magnets, a complex improvement in many aspects of tokamak magnet technology is necessary: enhancement of superconductor properties, improvements in structural materials, and stronger insulation.

More advanced diagnostics and quench protection may result in increased current density and improved reliability of the magnets. On the other hand, some of the problems of tokamak magnets may be relieved or become trivial with the availability of new HTC conductors [16], which are less sensitive to losses, ramp rate limitation, stability, and quench detection problems, and allow for easier cooling. Although highly speculative at the moment, there will be even more challenging tasks after the burning plasma experiment period which will take place perhaps 20-30 years from today. At this time it is expected that a DEMO reactor will be designed and built before any commercial fusion power plant is built. Assuming magnetic confinement will remain the most attractive fusion concept, at that stage the issues associated with radiation, such as long lifetime and low activation materials, will assume paramount importance on top of the problems outlined in this paper.

\section{REFERENCES}

[1] 2002 Fusion Snowmass Summer Study materials. Snowmass, CO, Next major steps in fusion, http://web.gat.com/snowmass/

[2] L.Waganer, 2002 Fusion Snowmass Summer Study, Cost assessment, unpublished.

[3] ITER Final Design Report, July 2001.

[4] V.D.Shafranov. Optimum shape of a toroidal solenoid, Sov. Phys.-Tech. Phys. 17,1433 (1973)

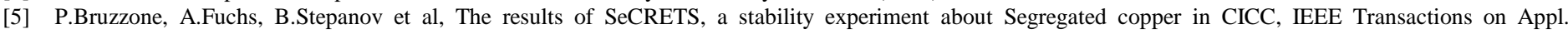
Superconductivity, v.11,No.1, March 2000, p.2018-2021.

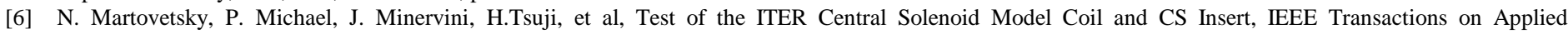
Superconductivity v.12, N 1 March 2002, p. 600-605

[7] N. Martovetsky, M. Takayasu, J. Minervini T. Isono,et al, Test of the ITER TF Insert and Central Solenoid Model Coil, presented at ASC 2002, Houston

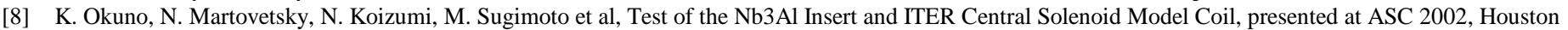

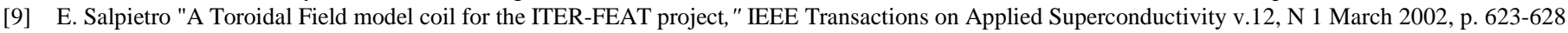

[10] R.Scanlan, D.Diedrich, Progress and plans for the US HEP Conductor development program, Presented at ASC 2002 , Houston, August 2002.

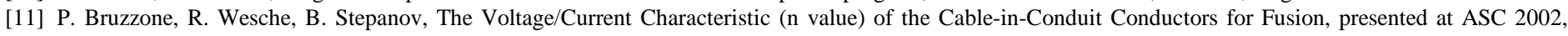
Houston, August 2002

[12] R.Scanlan, Conductor cost/performance status report for Snowmass 2001, unpublished

[13] D.Bessette, private communication

[14] D.Bessette, presentation at the US-Japan magnet technology for fusion workshop, MIT, August 2002.

[15] N.Mitchell, Proposal for Staged Strand Procurement for ITER Construction, ITER memo Aug. 28, 2002, unpublished

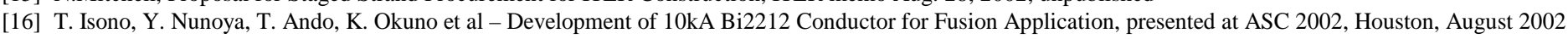

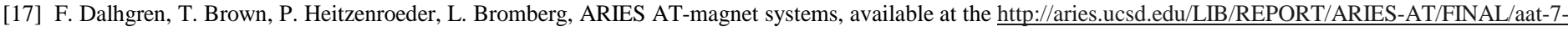
magnet.pdf

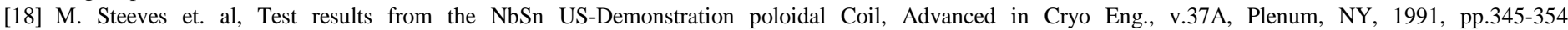

